\title{
A New Energy Efficient Clustering Protocol for Life Timemaximization in Wsns
}

\author{
Sribindu Sattu, Kumaraswamy Gajula, Ranjan Kumar Singh
}

\begin{abstract}
On this paper we have got proposed an energy inexperienced clustering protocol that is dynamic, distribution, self-organizing and further electricity green than gift protocols. Contributors in wi-fi sensor network clusters transport records to their cluster heads using TDMA scheduling. The cluster head transmits the aggregated and compressed packets to the bottom station. The CSMA MAC protocol is traditionally used for data transmission. In the CSMA MAC protocol, whilst the packet arrives on the cluster head it first senses the channel, if it's far loose, the cluster head transmits records that may be every a single packet or a a couple of packet. The cluster is reduced in transmitting unmarried packets from the pinnacle to the bottom station electricity. To make better utilization of sources along with energy more than one packets to be sent in area of transmitting single packet. On this proposed technique, we particularly focused on site visitors at the Cluster heads i.E. Cluster heads with big site visitors are desired for channel project and cluster head site traffic is a great deal much less for backoff time, in this era cluster head may also moreover collect greater records from their individuals. So on this manner strength overall performance is improved via who choose transmission of multiple packets in location of a single packet.In this proposed approach, Fuzzy C-way (FCM) set of rules is accompanied to form clusters inside WSNs. MATLAB software program is used to implement and evaluate the effects of the proposed set of rules with deterministic inexperienced clustering (DEC) set of regulations, Voronoi set of guidelines and LEACH protocol In terms of lifespan and electricity intake. The consequences show that the existence of the proposed algorithm is extended with the beneficial aid of $17 \%$.
\end{abstract}

\section{INTRODUCTION}

Advent-wi-fi sensor networks (WSNs) incorporate a massive massive type of sensor devices that could speak with others through wi-fi channels, with restricted electricity and computing abilities. Each sensor node is prepared with a transducer, microcomputer, transceiver, and electricity source. The transducer generates electric powered signs based totally totally on sensory physical consequences and occasions. The microcomputer tactics and shops the sensor output. The transceiver receives commands from a relevant laptop and transmits records to that computer. The strength of each sensor node is derived from an electric powered powered powered software program application or a battery. automation, healthcare tracking, surveillance and further. Sensor nodes have limited electricity, as quickly as they'll be

Revised Manuscript Received on September 10, 2019.

Sribindu Sattu, Associate Professor, CMR Institute of Technology,

Hyderabad, Telangana, India.

(Email: sribindu.sattu@gmail.com)

Kumaraswamy Gajula, Assistant Professor, CMR Institute of Technology, Hyderabad, Telangana, India.

(Email: kumaraswamygajula@gmail.com)

Ranjan Kumar Singh, Shri Ram College of Engineering \& Management, Palwal, Telangana, India.

(Email: ranjankumarsingh07@gmail.com).
Packages of WSN encompass aim monitoring, domestic

deployed they can't be recharge or update so energy conservation is considered the maximum critical function in WSNs. It is consequently vital to format powerinexperienced protocols for WSNs. Energy green networks are divided into groups to form nodes. Each cluster has a pacesetter node referred to as a cluster head $(\mathrm{CH})$. Cluster members ship information to their $\mathrm{CH}$ and $\mathrm{CH}$ deliver compressed and aggregated packet to base station. CSMA MAC The protocol is used for facts transmission. Within the CSMA MAC protocol, as fast as a node receives a packet this is to be despatched, it checks whether the channel is busy or no longer (no different node is transmitting at that element). If the channel is plain, the packet is despatched. If the channel isn't clear, the node waits for a randomly selected time, after which to look if the channel is apparent. This term is known as the backoff detail, and is counted through manner of using the backoff counter. If the channel is clear till the backoff counter is 0 , the node transmits the packet. If the channel isn't clean at the identical time because the backoff counter reaches 0 , the backoff problem is about all all over again, and the method is repeated.

Inside the CSMA MAC protocol, $\mathrm{CH}$ transmits facts that may be unmarried packets or more than one packets. In transmitting unmarried packet from $\mathrm{CH}$ to BS strength is decreased and to aggregate plenty less records strength is also reduced. A good way to make higher utilization of assets which include electricity more than one packets are favored for transmission. Inside the proposed method excessive precedence is given for information transmission to $\mathrm{CHs}$ having more packets at the same time as low precedence is given to CHs having fewer packets. Backoff time is extended for CHs having fewer packets, on this time $\mathrm{CH}$ may additionally collect statistics from extraordinary nodes after which it may get proper of access to the channel. This results in a higher community lifetime because of power financial savings for sensor nodes.

In this work, the fuzzy C-way (FCM) set of policies is found to shape clusters interior WSNs. This set of rules is a gentle segmentation approach that gives a diploma of cluster for every sensor node. Via imposing this set of regulations, it's miles predicted to conquer the problem of uneven distribution of sensor nodes associated with the software application of protocols which includes LEACH. Clusters are businesses of sensor nodes which might be fashioned on this shape of way that the complete spatial distance among sensor nodes within each cluster is minimized. To begin with cluster heads are constructed the use of FCM. The cluster head roll is grew to become round among nodes to 
keep away from speedy drainage of nodes' electricity. The cluster head roll is grew to become around based totally totally on residual power.

The rest of the paper is ready as follows. The evaluate of previously proposed algorithms is in section II. In section III, we talk the preliminaries. In segment IV problem method and proposed set of hints is noted. In phase $\mathrm{V}$ we display the experimental consequences and take a look at the only-of-a-type algorithms. In the end, in phase VI, we end the paper.

\section{RELATED PAINTINGS}

In wi-fi sensor networks are famous technologies with precise features associated with cluster-based totally completely protocol scalability and inexperienced communication. The nodes are divided into groups and with a frontrunner node i.E. Cluster head $(\mathrm{CH})$. $\mathrm{CH}$ performs information aggregation and fusion techniques for inexperienced use of energy assets. In this way the overall quantity of facts being transmitted to BS is drastically decreased. The concept of hierarchical routing is also used to perform power-green routing in WSNs. Severa cluster primarily based protocols have been proposed with the resource of severa researchers. The Low power Adaptive Clustering Hierarchy (LEACH) is an ordinary cluster-based totally protocol using a allotted cluster set of rules. LEACH cluster heads are generated randomly because of this because of the fact some nodes are located at the threshold of the cluster. LEACH is best a probabilistic method in which the range of cluster heads is not consistent.

Other cluster-based without a doubt protocols based totally on LEACH are PEGASIS, teen, APTEEN and HEED. But, those protocols decorate community lifetime via growing the performance of data transmission, however the cluster shape isn't optimized.

In the international community the site visitors load among CHS is balanced and the strength consumption a number of the sensor nodes inside the community cluster is balanced.

The FCM algorithm has been achieved in WSNs and tested to beautify network normal typical performance in terms of energy conservation while the usage of some conventional techniques which includes direct conversation, minimal transmission strength, LEACH and adequatemanner and so on. Are provided within the works. 1,2]. Cluster based totally completely multichannel tool the usage of mobile shape has been finished in [3]. Most beneficial sensor nodes deployment using FCM and most important wide form of clusters the usage of FCM are supplied in [45]. An power green cluster primarily based without a doubt protocol the usage of FCM set of policies has been completed in [6]. To noticeably optimize strength and communication overall performance in clusters, multichannel structures can be used because it reduces radio interference, opposition to get entry to wireless channels, and bandwidth business corporation [7]. As an instance, we are capable of use a mobile community technique that offers one frequency channel consistent with cluster, respecting the reuse of the spatial channel. So, all radio transceivers within a cluster perform at the identical frequency channel.
[8] the author gives a deterministic approach, which determines that a difficult and speedy range of cluster heads are decided on consistent with round. The DEC proves to be extra robust and further strong than the hazard-based completely totally fashions. The DEC setup section is modified in LEACH, however maintain the everydaykingdom phase the identical. For the cause that electricity of a node may be determined a priori, the $\mathrm{CH}$ election method is reorganized to use best the residual energy ( $\mathrm{RE}$ ) of each node.In DEC, inside the early levels, BS chooses at-cluster heads for the network. Setup segment of DEC is huge.

The Voronoi diagram is a famous computational geometry shape, frequently used in WSNs for coverage troubles and to discover remarkable clusters. It divides a given location into numerous Voronoi cells in this type of way that high-quality one sensor is discovered in each cellular. The Voronoi diagram can function a critical sampling method for WSN coverage, with sensors appearing as websites. If the whole Voronoi polygon vertical is covered then the location is surely covered.

In each cell, in location of each special sensor cell in a given subject, all points are near the sensor in it. Do not forget sensors P1 and P2 inside the given plane. A bisector is drawn perpendicular to the road section connecting the 2 sensors from the Voronoi cellular. A triangle with vertices $(\mathrm{P} 1, \mathrm{P} 2$, and $\mathrm{X})$ is constructed on the primary 3 places, with the zero. 33 sensor $X$ to acquire a Voronoi diagram for the three internet web sites. Then the bisectors perpendicular to the 3 facets of a triangle are drawn to fulfill at a factor. The Voronoi set of guidelines is a totally complicated set of policies.

\section{PRELIMINARIES}

We rst gift the assumptions and model of the community under attention.

\section{A. Assumptions-}

We remember scenario of utility in which sensor nodes are deployed randomly on the way to constantly show the surroundings. The data amassed by means of manner of the use of sensor nodes is sent to a base station located a long way from the deployment eld.

Moreover, other assumptions are made as look at:

- Sensor nodes similarly to base station are desk sure after being deployed within the eld.

- The network is considered homogeneous and all the sensor nodes have the same preliminary strength.

- each sensor node is aware about their personal geographical feature.

- All nodes degree the environmental parameters at a xed price and ship it periodically to the receiver nodes.

- The radio channel is symmetric such that power consumption of information transmission from node $\mathrm{A}$ to node $B$ is just like that of transmission from node B to node A. 
Each sensor nodes can feature each in sensing mode to display the surroundings parameters and transmit to the bottom station or cluster head mode to collect information, compress it and in advance to the BS.

\section{B. Radio power fashions -}

As citing above, in most cases of application, among its components eating energy which include statistics processing unit, sensing unit, memory storage and transceiver, the radio communique unit takes the maximum energy expenditure. Therefore, we particularly bear in mind the strength consumption of the sensor nodes for communique inside the community. Sensor node critical additives and associated power intake are tested in Fig.1.

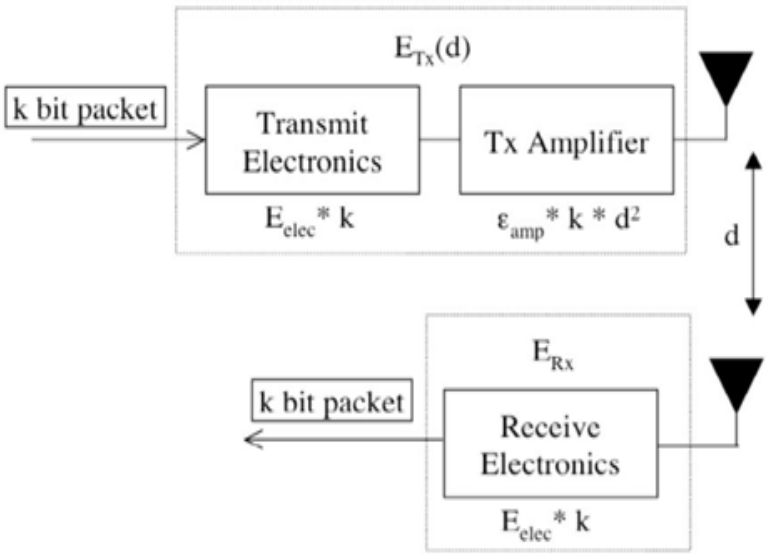

Figure 1: Radio energy model

Both the loose area and multipath fading channel models in [9] are used to compute energy dissipated in the course of the system of transmitting and receiving information. The power intake for transmitting 1 bit message over a distance $\mathrm{d}$ is

$$
\begin{gathered}
E_{T x}=E_{\text {slec }} * l+E_{f s} * l * d^{2} \\
E_{T x}=E_{\text {elec }} * l+E_{f s} * l * d^{2}, \mathrm{~d}<d_{0} d_{0} \\
E_{T x}=E_{\text {elec }} * l+E_{m p} * l * d^{4} \\
E_{T x}=E_{\text {elec }} * l+E_{m p} * l * d^{4}, \mathrm{~d} \geq d_{0} d_{0}
\end{gathered}
$$

and for receiving this message respectively is:

$$
E_{R x}=E_{\text {elec }} * l E_{R x}=E_{\text {elec }} * l
$$

where $E_{\text {elec }}$ Is the strength spent to perform the transceiver circuit, Efs and Emp are the strength expenditure of transmitting one bit records to acquire a appropriate bit mistakes rate and is relying on the space of transmission within the case of free space model and multipath fading version. If the transmission distance is less than a threshold, the free space model is carried out; otherwise, we use the multipath model. the edge is calculated as-

$$
d_{0} d_{0=} \sqrt{E_{f s} / E_{m p}} \sqrt{E_{f s} / E_{m p}}
$$

Another parameter is also taken into account is the data aggregation energy expenditure which is set as $\mathrm{Eda}=5 \mathrm{~nJ} / \mathrm{bit} / \mathrm{message}$. We use the following values for other parameters: Eelec $=50 \mathrm{~nJ} / \mathrm{bit}, \mathrm{Efs}=10 \mathrm{pJ} / \mathrm{bit} / \mathrm{m}^{2} \mathrm{~m}^{2}$, and $\mathrm{E}=$ $0.0013 \mathrm{pJ} / \mathrm{bit} / \mathrm{m} 4$.

Considering $\mathrm{N}$ node network partitioned into c clusters, the average number of nodes in cluster is $\mathrm{N} / \mathrm{c}$, the energy consumption of the $\mathrm{CH}$ to receive message from the non cluster head is

$$
E_{R x-C H} E_{R x-C H}=l E_{\theta l e c}\left(\frac{N}{c}-1\right) l E_{\theta l e c}\left(\frac{N}{c}-1\right)
$$

and to aggregate data into a l-bit message and send it to the $\mathrm{BS}$ is

$$
\begin{gathered}
E_{T x-C H} E_{T x-C H}=l E_{d a} \frac{N}{c} l E_{d a} \frac{N}{c} \\
l E_{\theta l e c}+l E_{m p} d_{t o B S}^{4} l E_{\theta l e c}+l E_{m p} d_{t o B S}^{4}
\end{gathered}
$$

where $d_{\text {toBS }} d_{\text {toBS }}$ is the average distance from one $\mathrm{CH}$ to the BS. Assuming that the distance from the non cluster head node and the $\mathrm{CH}$ is short, the energy consumed by the non-cluster head node to transmit a $l$-bit message is

$$
\begin{aligned}
& E_{T x-\text { nonCH }} E_{T x-n o n C H}=l E_{\text {elec }}+l E_{f s} d_{\text {toCH }}^{2} \\
& l E_{\text {elec }}+l E_{f s} d_{\text {toCH }}^{2} \\
& \text { where } d_{\text {to } C H} d_{\text {toCH }} \approx \frac{1}{2 \pi} \frac{M^{2} 1}{c 2 \pi} \frac{M^{2}}{c} \text { which is the average }
\end{aligned}
$$
distance from one node to its $\mathrm{CH}$ and $\mathrm{M}$ is the network diameter. Thus, the total energy dissipated in the network during a round of collecting data and transmitting to BS is

$$
\begin{aligned}
& E_{\text {round }} E_{\text {round }}= \\
& l\left(2 N E_{\text {elec }}+N E_{d a}+c E_{m p} d_{\text {to } B S}^{4}+N E_{f s} d_{\text {to } C H}^{2}\right) \\
& l\left(2 N E_{\text {elec }}+N E_{d a}+c E_{m p} d_{\text {toBS }}^{4}+N E_{f s} d_{\text {to } C H}^{2}\right) \\
& \quad= \\
& l\left(2 N E_{\text {elec }}+N E_{D A}+c E_{m p} d_{\text {toBS }}^{2}+N E_{f s} \frac{1}{2 \pi} \frac{M^{2}}{c}\right) \\
& l\left(2 N E_{\text {elec }}+N E_{D A}+c E_{m p} d_{\text {toBS }}^{2}+N E_{f s} \frac{1}{2 \pi} \frac{M^{2}}{c}\right)
\end{aligned}
$$

This tremendous strength expenditure consists of the common power dissipated through facts transmission of non-cluster head nodes and $\mathrm{CHs}$ and the energy consumption for facts collection and fusion of the CHs.

\section{Network model-}

We discover that sensor nodes are randomly deployed to discover, tune and transmit / relay positive bodily sports to the cease-patron through the sync network. On this paintings, the bushy C-manner (FCM) set of regulations is accompanied to form clusters inside WSNs. We take into account the 2-tiered heterogeneous WSN illustrated in determine 1. Wherein all sensor nodes at degree 1 communicate with their $\mathrm{CH}$ and characteristic on 802.15.4 channels. The diploma 2 sink node $(\mathrm{CH})$ communicates at the bottom station and operates on a unmarried 802.Eleven channel. All radio transceivers in a degree 1 community have the identical radio variety. We expect that sink nodes are less strength-constrained and feature radio transceivers: one for degree 1 and the other for degree 2. To reduce radio interference between clusters multichannel tool is used Multichannel system optimized strength and verbal 
exchange overall performance. For the aggregate of multichannel structures for our clustering protocol, we anticipate that simplest the sinks recognize their frequency channel $\mathrm{Cx}$ if you want to be assigned to their cluster. To have considered one of a kind intra-cluster verbal exchange sensor nodes of the equal cluster, switch your radio transceiver to a devoted frequency channel for the cluster tested on figure 2. Four 802.15.Four frequency channels (Cx) are enough to create a pattern and facilitate reuse of the spatial channel.

802.11 links

Sink node Sensor node CxChannel allocated to the cluster $\mathrm{r}$

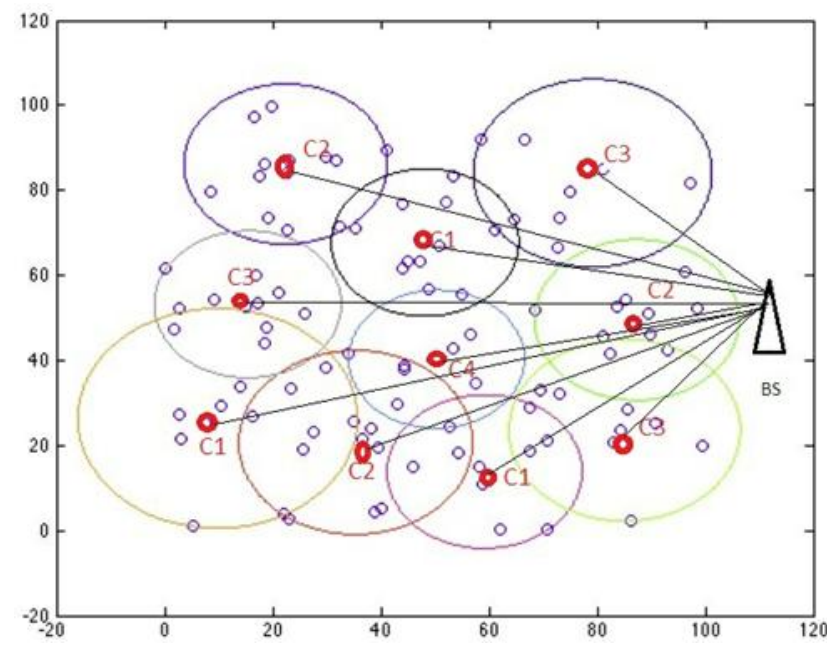

Figure 2.Proposed topology inside a large-scale WSN with different frequency channels.

\section{D.Fuji C MEANS algorithm-}

Fuzzy C- (FCM) is a method of clustering that allows a piece of data to belong to two or more groups. This method (developed by Dunn in 1973 and improved by Bezdek in 1981) is often used in pattern recognition.

Let $\mathrm{Xi}$ is the number of sensor nodes where $\mathrm{i}=1,2, \ldots \ldots \ldots \mathrm{N}$ and $\mathrm{C} \mathrm{j}$ is the number of cluster heads where $\mathrm{j}=1,2, \ldots \ldots . \mathrm{C}$

It is based on minimization of the following objective function:

$$
\sum_{i=1}^{N} \sum_{j=1}^{C} \mu_{i j}^{m} d_{i j}^{2}
$$

$$
J_{m=}^{J_{m}} \sum_{i=1}^{N} \sum_{j=1}^{C} \mu_{i j}^{m} d_{i j}^{2}
$$

$\mu_{i j} \mu_{i j}$ is node i's diploma of belonging to cluster $\mathrm{j}$, dij is the Euclidean distance among node $i$ and the center factor of cluster $\mathrm{j}$. wherein $\mathrm{m}$ is the fuzzification parameter that is any actual wide variety extra than 1 .

Fuzzy partitioning is performed thru an iterative optimization of the intention feature shown above, with the update of membership $\mu_{i j} \mu_{i j}$ and the cluster centers $C_{j} C_{j}$ by:

$$
C_{j} C_{j}=\frac{\sum_{i=1}^{N} \mu_{i j X_{i}}^{m} \sum_{i=1}^{N} \mu_{i j X_{i}}^{m}}{\sum_{i=1}^{N} \mu_{i j}^{m} \sum_{i=1}^{N} \mu_{i j}^{m}}
$$

The degree $\mu_{i j} \mu_{i j}$ of node $\mathrm{i}$ respected to cluster $\mathrm{j}$ is calculated by:

$$
\mu_{i j} \mu_{i j=} \frac{1}{\sum_{k=1}^{c}\left(\frac{d_{i j}}{d_{k j}}\right)^{2 /(m-1)} \sum_{k=1}^{c}\left(\frac{d_{i j}}{d_{k j}}\right)^{2 /(m-1)}}
$$

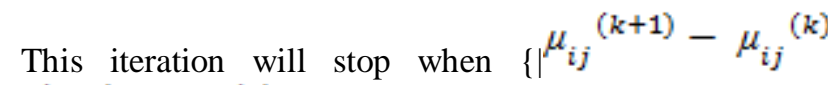
$\left.\mu_{i j}{ }^{(k+1)}-\mu_{i j}{ }^{(k)} \mid\right\}<\varepsilon \varepsilon$, where $\varepsilon \varepsilon$ is a termination criterion between 0 and 1 , Where $\mathrm{k}$ are the iteration steps. This procedure converges to a minimum value of $J_{m} J_{m}$..

\section{PROBLEM ADDITIVES AND PROPOSED SET OF RULES}

For information transmission CSMA MAC protocol is desired. In CSMA, as soon as a node gets a packet that is to be despatched, it checks the channel is busy or no longer (no one-of-a-kind node is transmitting at the time). If the channel is apparent, then the packet is despatched. If the channel isn't always clean, the node waits for a randomly selected time period, after which tests yet again to appearance if the channel is apparent. This time body is known as the backoff aspect, and is counted down by using a backoff counter. If the channel is apparent on the identical time as the backoff counter reaches zero, the node transmits the packet. If the channel isn't clean at the identical time because the backoff counter reaches 0 , the backoff element is set all all over again, and the system is repeated. In CSMA, CH transmits information which can be unmarried packet or multiple packets. In transmitting unmarried packet from $\mathrm{CH}$ to $\mathrm{BS}$ strength is reduced. To make better utilization of belongings on the facet of energy multiple packets to be despatched rather than transmitting unmarried packet.

We advocate an strength-inexperienced Clustering protocol this is primarily based absolutely mostly on CSMA MAC protocol.

\section{A. Clustering calculation}

We recollect that $\mathrm{N}$ sensor nodes are deployed randomly proper right into a eld with an area of MxM . After being spread out, these sensor nodes ship a message to the lowest station with the data in their geographical place. Based totally totally on this statistics the lowest station will calculate the cluster facilities and allocated sensor nodes into cluster the use of FCM set of rules. In the case of our software, FCM set of rules is executed to cluster the sensor nodes. Every node is assigned a degree of belonging to cluster head rather than clearly being a member of simply one cluster. Therefore, the nodes close to the boundary of a cluster can also become humans of the cluster with a diploma approximating the diploma of belonging to the neighbor clusters. The calculation is completed with the aid of way of the above FCM set of policies. The convergence is completed whilst the distinction a number of the 
coefficients in iterations is a good deal a good deal much less than a threshold or a massive range of iterations is reached. As soon as the cluster introduction is entire, base station supply the statistics of the cluster head and to which cluster a node belongs to all of the nodes. Some nodes are overlapped within the clusters. In our approach overlapped nodes are associated with $\mathrm{CH}$ having better degree of club.

A multi-channel tool is used to create a mobile form thru assigning one frequency channel

Consistent with cluster. To differintracluster communication amongst clusters and to lessen radio interference frequency reuse concept is used. In which at degree 1 all sensor nodes talk to their $\mathrm{CH}$ the use of 802.15.4 channels which operates at the 2.Four $\mathrm{GHz}$ frequency bands with a statistics rate of $250 \mathrm{~Kb} / \mathrm{s}$, a radio form of 50 meters. At stage 2 sink node $(\mathrm{CH})$ communicate to base station the usage of unmarried 802.Eleven channel massive with a statistics fee of $1 \mathrm{Mb} / \mathrm{s}$, a radio variety of 500 m. In our approach we use four channels for clusters. It reduces the competition to access the wi-fi channel and the bandwidth career.

Steps observed in FCM set of rules:

Input : $\mathrm{X}$ dataset

Output: $\mathrm{C}$ clusters

Step 1: Generate N nodes randomly

Step 2: select C clusters randomly

Step 3: find out centroid the use of FCM set of guidelines

Step four: find club charge the use of FCM set of guidelines from c clusters to all nodes and store in a matrix $\mathrm{U}=[\mathrm{]}$

Step five: update club costget admission to

Else watch for $\mathrm{t} \mu \mathrm{s} \mathrm{CH}$ gathers facts from CMs in $\mathrm{t} \mu$ s or after $t \mu$ s remove Get channel get right of entry to

Prevent

Wherein $\Delta$ pkt is threshold packet and $\mathrm{t} \mu \mathrm{s}$ is backoff length.

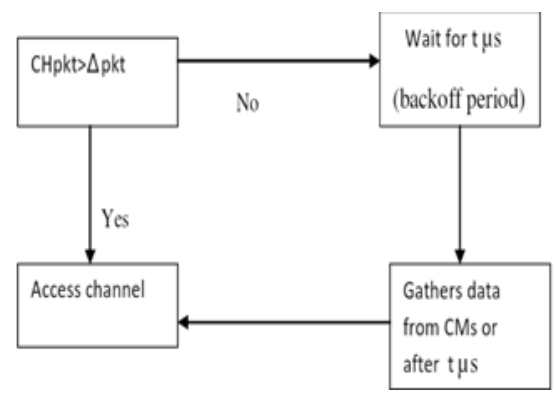

Figure3: Proposed algorithm

\section{SIMULATION RESULTS}

Matlab software program program is used as simulation platform to simulate proposed set of guidelines, DEC set of policies, Voronoi diagram and LEACH protocol. The proposed set of guidelines hobbies at balancing the entire power consumption of nodes and developing the community's lifetime. So we degree proposed set of guidelines performance from two factors: the lifetime and the whole power consumption of the network. The existence of community approach the time from the start of simulation to the time at the same time as the last node died. As the electricity of WSN is restricted, so the strength consumption in its lifetime is a top notch indicator to degree the overall normal overall performance of it.
For the motive of benchmarking our protocol in opposition to others within the literature, we chose to check in competition to a few well known proposals, LEACH protocol, Voronoi diagram and a extra trendy one, DEC. The ones protocols belong to two specific techniques of clustering; the LEACH is a stochastic clustering scheme and makes a smart use of dynamic thresholding, whereas the DEC is a in easy terms deterministic protocol in which regular numbers of $\mathrm{CHs}$ are elected consistent with round. These protocols are accurate baseline for evaluation due to the following skills:

- Clustering is despatched and sincerely based completely totally on network data.

- Clusters are disjoint.

- they are designed to gather power efficiency via load balancing.

- every employs a completely specific mode of $\mathrm{CH}$ election.

Those are the overall overall performance metrics applied in our investigations:

- power normal with round: This metric represents the power dissipated via all nodes in a round of data

\section{COLLECTION.}

- network lifetime: This metric represents the time period from the instantaneous the network is deployed to the immediate even as the primary node runs out of electricity

Simulation parameters-

For the ones simulation experiments, we assumed that there are 100 sensor nodes dispensed randomly in a square $\mathrm{M} \times \mathrm{M}$ place with $\mathrm{M}=100 \mathrm{~m}$. The transceiver energy parameters are set as: $=50 \mathrm{~nJ} / \mathrm{bit}$ and $=10 \mathrm{pJ} / \mathrm{bit} /$. The electricity for records aggregation is ready to $=$ five $\mathrm{nJ} / \mathrm{bit}$ in line with signal. The manage and records message sizes are consistent 6400 bits.

\begin{tabular}{l|l|}
\hline Parameters & Values \\
\hline Network size & $\left(100 \mathrm{X} 100 \mathrm{~m}^{2} \mathrm{~m}^{2}\right.$ \\
\hline No of nodes & 100 \\
\hline Initial energy & 0.1 joules \\
\hline Packet size & $6400 \mathrm{bits}$ \\
\hline$E_{\text {elec }}$ & $50 \mathrm{nj}$ \\
\hline$E_{f s}$ & $10 \mathrm{pj}$ \\
\hline$E_{m p}$ & $1.3 \times 10^{-15} 10^{-15}$ \\
\hline$E_{R X}$ & $E_{\text {elec }} E_{\text {elec }} * k$ \\
\hline$E_{D A}$ & $5 \mathrm{nj}$ \\
\hline
\end{tabular}

\section{A. The community lifetime}

The network lifetime is described because the time from the start of the simulation to the time at the same time as the final node died. In WSN, the community life is cut up into solid and unstable period. Solid period normally method the time from the begin of the simulation to the time while the number one node dies, the volatile duration refers to the time from the lack of lifestyles of first node to the surrender of simulation. 
If it occurred that a few nodes begin to die, the network operation may additionally moreover end up risky and unreliable facts moving will arise. Therefore, the longer the stable length is, the better the general overall overall performance of the community. In clustering, cluster heads are responsible not splendid for speakme with the bottom station, however for the data fusion. Randomly shelling out the nodes and randomly selecting the cluster heads motives a few cluster heads die in advance because of the low energy or the extended distance to base station. Secondary cluster heads are set for the ones clusters to be accountable for the verbal exchange with not unusual nodes and statistics fusion, this balances the energy load of cluster heads and avoids premature loss of life of those cluster heads, so the sturdy length of network lifetime might be extended.

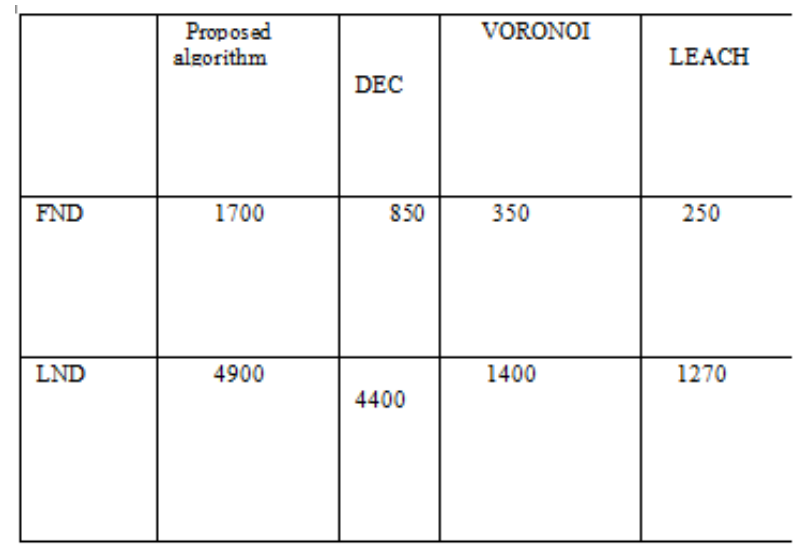

Table 2: first node died and last node died of different algorithms

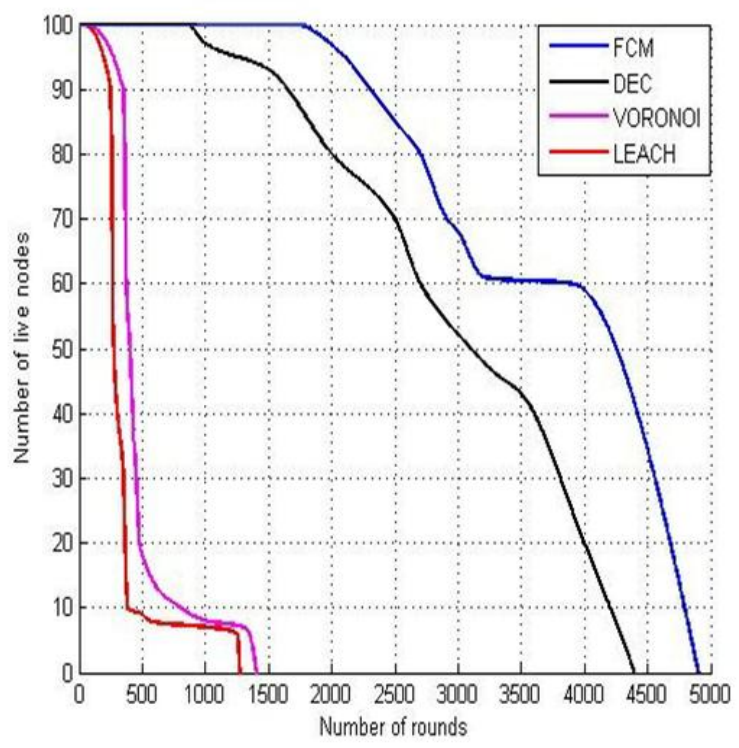

Discern four: quantity of live nodes constant with spherical

Discern four shows the network lifetime, simulation outcomes shows that the community life of the proposed set of rules is more than exclusive protocols. When ninety\% nodes died, the network reliability is surprisingly reduced and the taking walks is almost meaningless. Network lifetime is described because of the truth the time from the simulation beginning to the time $90 \%$ nodes died as powerful lifecycle, analysing from figure four, we understand that the powerful lifecycle of the progressed set of pointers is longer $10 \%$ than that of DEC protocol. The proportion of robust period of lifecycle of DEC set of policies is $17 \%$, balance inside the improved protocol is $34 \%$, the proportion of stable duration of lifecycle in progressed set of policies will growth through the usage of $17 \%$. This shows that the strolling performance of stepped forward protocol is a superb deal better than that of various Protocols.

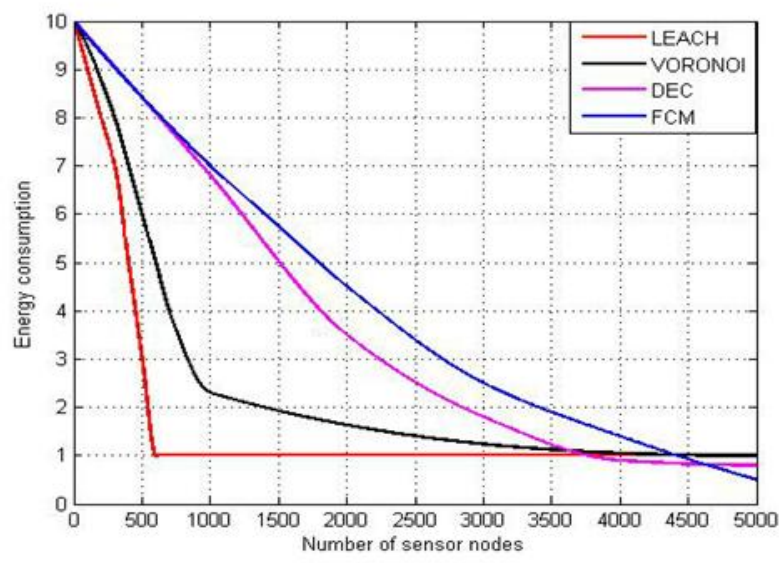

Determine 5: electricity intake steady with spherical

\section{B. The whole power intake}

parent five is the strength intake curve. Improved set of regulations reduced the power intake of few cluster heads which has low strength or is a protracted way away to base station with the resource of placing secondary cluster heads pretty. This balanced the electricity intake of the entire network, extended the lifestyles of cluster heads which might also moreover die earlier and optimized the general overall performance of the network

Thereby decreased the whole energy consumption of the effective lifecycle. From the assessment of determine 5, we understand that in the entire strolling of the network, the strength intake of improved set of policies is a lot decrease than that of various Protocols on the equal round of simulation. These consequences are regular with the layout functions of advanced set of rules.

Parent 6 showing as range of nodes will boom lifetime of the network moreover increases and power intake decreases (figure7). Determine 8 displaying as network size will boom power consumption will growth however lifetime decreases (determine nine). This decide shows proposed set of policies is scalable. Those graphs show that our proposed set of suggestions outperforms great. 


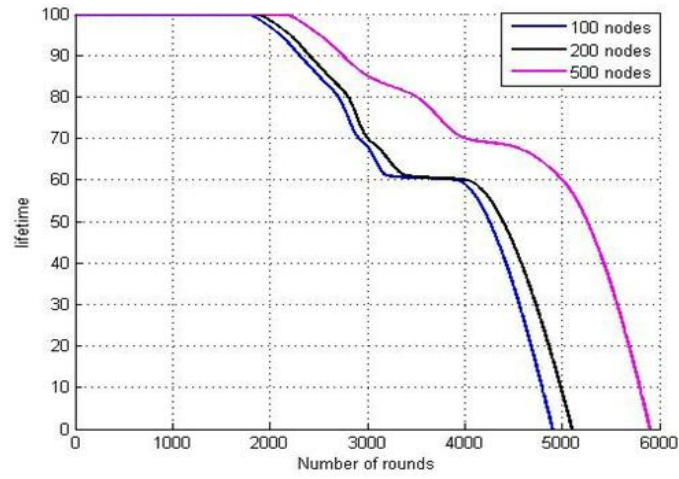

Figure 6: Network lifetime of proposed algorithm with 100,200 and 500 nodes

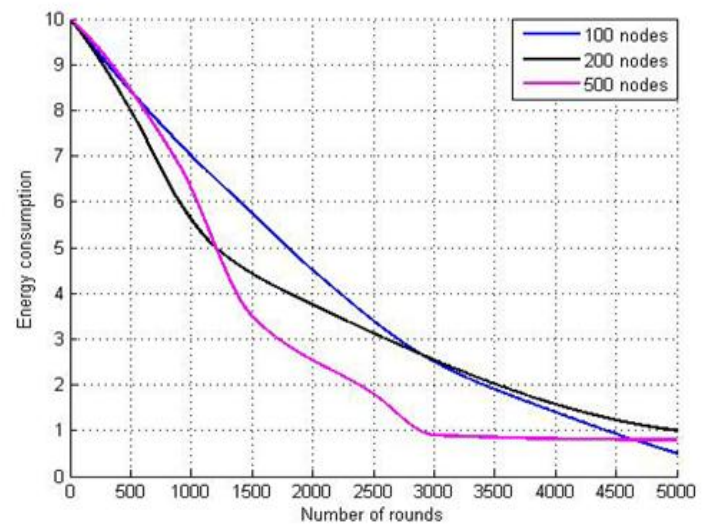

Figure 7: Energy consumption of proposed algorithm with 100,200 and 500 nodes

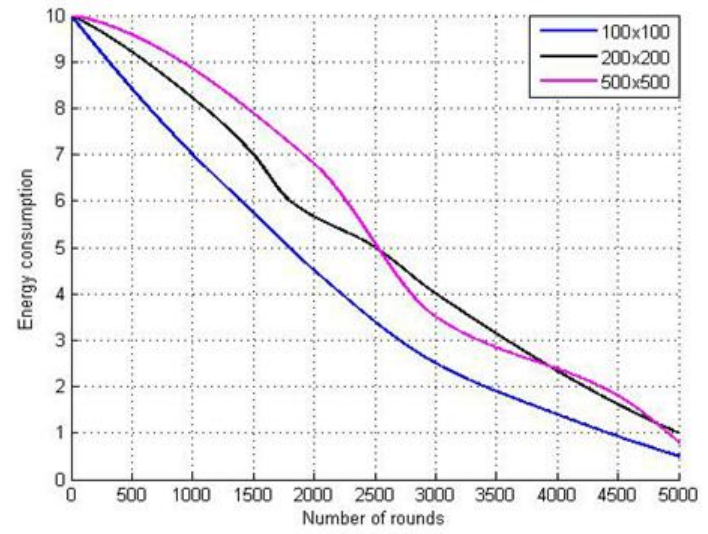

Figure 8:Energy consumption of proposed algorithm for different field size

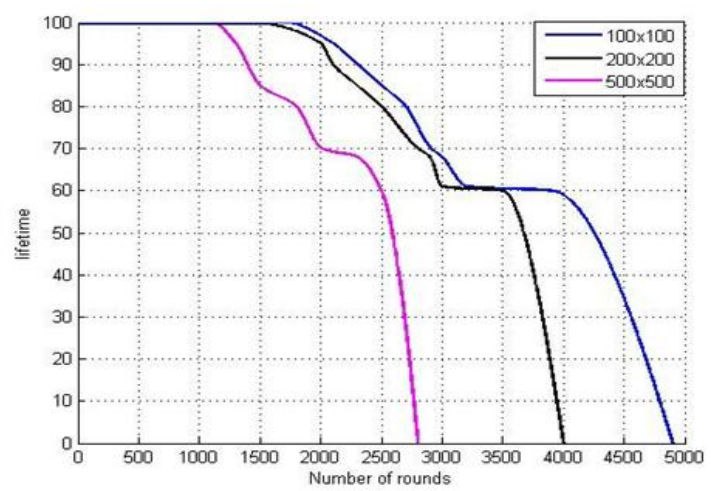

Determine 9: community life of proposed
Set of rules for one-of-a-type subject duration

.VI. Cease - in this paper we present a singular set of regulations to decorate the life of the community this is based totally on CSMA MAC protocol. FCM clustering protocol is used for clustering the nodes. We have proposed a extremely good way for information transmission. In CSMA, CH transmits facts to the lowest station which can be single packet or multiple packets. In transmitting unmarried packet from $\mathrm{CH}$ to $\mathrm{BS}$ energy is decreased. To make higher utilization of belongings along side electricity a couple of packets to be despatched in desire to transmitting unmarried packet. In proposed set of rules, high priority is given to $\mathrm{CH}$ having large packet than $\mathrm{CH}$ having a lot less packet for channel mission. To make energy green community backoff length is multiplied of $\mathrm{CHs}$ having much less data in this era they will gather extra information from their cluster contributors and can get right of entry to the channel. Our simulation effects shows that through using utilizing proposed set of policies the energy intake is reduced and the life time of the network is extended through $17 \%$ at the same time as compared with LEACH, Voronoi and DEC protocols. The difficulty of this protocol is that it isn't applicable in put off touchy programs.

\section{REFERENCES}

1. Dasgupta, S., Dutta, P.: 'An progressed leach method for head desire technique in a fuzzy-C manner introduced approximately clustering of a wi-fi sensor network'. Proc. IEMCON 2011 Organised via using manner of IEM in Collaboration with IEEE, five and 6 January 2011, pp. 203-208

2. Singh, A.Ok., Goutele, S., Verma, S., Purohit, N.: 'An energy green approach for clustering in WSN using fuzzy common revel in', Int. J. Comput. Appl., 2012, forty four, (18), pp. Eight-12

3. C.T.Kone, M. David, F. Lepage : 'Cluster-primarily based absolutely multi-channel for boosting overall performance of big scale wireless sensor networks'.IEEE 2010,V3 163-168

4. Y. Good enough. Park, M. G. Lee, ok. Ok. Jung, J. J. Yoo: 'maximum first-class sensor nodesdeployement the use of FCM set of regulations', global symposium on pc technological records and society, 2011, pp. 389-392

5. A. S. Raghuvanshi, S Tiwari, R Tripathi , N. Kishore : 'top-best form of clusters in wireless sensor networks: an FCM technique'.IEEE 2010, pp. 817-823

6. D. C. Hoang, Rasjesh Kumar, Sanjib Kumar Panda: 'interest of a cluster based totally totally protocol the use of FCM set of recommendations for WSNs'.IETDL 2013,pp. 163-171

7. X. Wang, and T. Berger, "Spatial channel reuse in wi-fi sensor networks," Wirel. Netw.14, 2, March. 2008, pp. 133-146, doi:10.1007/s11276-006-8743-z

8. Femi A. Aderohunmu, Jeremiah D. Deng, Martin k. Purvis, "A Deterministic strength efcient Clustering Protocol for wireless Sensor Networks", IEEE 2011.

9. W. B. Heinzelman, A. P. Chandrakasan, and H. Balakrishnan, An software program-specific protocol shape for wireless microsensor networks, IEEE Transactions on wi-fi Communications, vol. 1, pp.660670, 2002. 